\title{
ASYMPTOTIC PERFORMANCE OF FIXED AND DYNAMIC CHANNEL ASSIGNMENT IN CELLULAR RADIO
}

Robert J. McElifit

Department of Electrical Engineering

California Institute of Technology

Pasadena, CA 91125
Lumar N. Sivarajan

IBM T. J. Watson Research Center

P. O. Box 704

Yorktown Heights, NY 10.598
We represent a cellular system by an undirected graph (withou1 loops or multiple edges) where the vertices of the graph represent the cells of the system and pairs of cells that are forbidden from using the same channel are joined by an edge. A channel is a frequency in the case of FDMA systems and a time slot in the case of TDMA systems. Two examples of cellular systems are shown in the figure.

We assume that the call arrivals in a cell are independent of call arrivals in all other cells and obey a Poisson distribution that the rate of arrivals is the same in every cell (uniform traffic). that the call-durations are exponentially distributed. and that a call does not leave a cell when it is in progress (no handoffs). Let $N$ be the number of cells and $C$ the number of available channels in the system. Let $\rho$ be the offered traffic per cell (measured in Erlangs). For any channel assignment scheme, let $T(\rho)$ be the carried traffic in the system and $M$ the maximum number of calls that can be in progress simultaneously in the system.

A fixed channel assignment (FCA) assigns a fixed number of channels permanently to each cell under the constraint that two cells joined by an edge are not assigned the same channel. A new call in a cell is assigned one of the channels assigned to that cell, if such a channel is available. Otherwise the call is blocked. A cell is not allowed to borrow a channel from another cell even if the other cell is not using that channel. An FCA can be represented by an $N$-vector of integers, $\left(c_{i}\right)$, where $c_{i}$ is the number of channels assigned to the $i$ th cell. Clearly, the maximum number of calls that can be progress is $M=\sum_{i} c_{i}$. For example, the $(1,1,1)$-FCA in the cellular system represented by G1 assigns one channel to each of the cells $(M=3)$ whereas the $(2,0,2)$-FCA assigns two channels to cells 1 and 3 and none to cell $2(M=4)$. Note that both of these fixed channel assignments require a total of 2 channels $(C=2)$.

In dynamic channel assignment, all the channels are available in all the cells and channels are assigned to the calls as they arise under the constraint that two cells joined by an edge are not assigned the same channel simultaneously. The Maximum Packing (MP) strategy [1] is a dynamic channel assignment strategy that allows the channels assigned to the calls in progress to be changed if it allow's a new call to be assigned a channel. If no channel can be found in spite of this, the call is blocked: otherwise the call is accepted. One might expect that MP will be better, i.e., have a higher carried traffic, than any FCA for the same value of $\rho$. This is true if no channel may be reused in the cellular system, i.e., if the cellular system is represented by a complete graph. (This phenomenon, which is basically a law of large numbers, is usually termed "trunking efficiency" in telephony.) It can also be shown that for any cellular system. MP has a higher carried traffic than FCA for sufficiently small $\rho$. However, surprisingly this is not always true.

It was shown in [1] that, for the infinite cellular system represented by G2, when $C=2$, the $(\ldots, 1,1,1, \ldots)$-FCA has a higher carried traffic than MP, for large $\rho$. This was the first

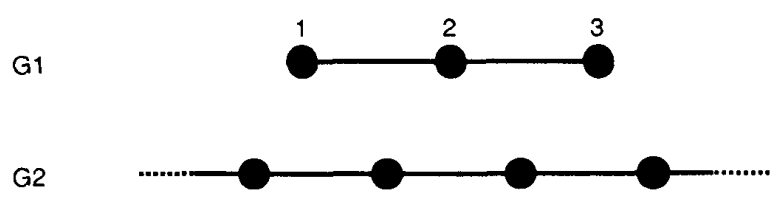

example of a cellular system where an FCA has a higher carried traffic than MP. In this paper, we study this interesting phenomenon further.

Theorem 1. For any channel assignment scheme.

$$
T(\rho)=M-\frac{\Pi_{M-1}}{\Pi_{M}} \frac{1}{\rho}+\frac{\Pi_{M-1}^{2}-2 \Pi_{M-2} \Pi_{M}}{\Pi_{M}^{2}} \frac{1}{\rho^{2}}+O\left(\frac{1}{\rho^{3}}\right) .
$$

for large $\rho$, where $\Pi_{i}$ is the probability that $i$ calls are in progress in the system.

Let $p_{i}=\Pi_{i} \Pi_{0}$. Then, $p_{i}$ may be substituted for $\Pi_{i}$ in the above result. This is important because, even for small values of $N$ and $C$ '. the required values of $\Pi_{i}$ are much more difficult to compute than the corresponding $p_{i}$.

Corollary 1. A $\left(c_{1}, c_{2} \ldots, c_{N}\right)$ - FCA satisfies $T(\rho)=M-$ $M / \rho+O\left(1 / \rho^{2}\right)$ for large $\rho$, where $M=\sum_{i=1}^{N} c_{i}$.

If $a$ denotes the largest number of cells that can simultaneously use the same channel ( $\alpha$ is the independence or stability number of the corresponding graph). it can be shown that $M \leq C \alpha$ for any channel assignment scheme. By the definition of $\alpha$ there is a set of a cells all of which may use the same channel. An FCA that assigns all $C$ available channels to each cell in this set achieves $M=C a$. The same argument shows that $M=C \alpha$ for MP.

Example 1. Consider the cellular system represented by the graph G1 and let $C=2$. The $(1,1,1)$-FCA satisfies (using Corollary 1) $T(\rho)=3-3 / \rho+O\left(1 / \rho^{2}\right)$ for large $\rho$. But, for large $\rho$ the $(2,0.2)$-FCA which satisfies $T(\rho)=4-4 / \rho+O\left(1 / \rho^{2}\right)$ has a higher carried traffic (and hence a lower blocking probability). In fact, its carried traffic is even higher than that of MP (using the same number of channels viz. $C=2$ ) which satisfies $T(\rho)=$ $4-8 / \rho+O\left(1 / \rho^{2}\right)$. So this is another example where MP has a lower carried traffic than an FCA for heavy traffic - but the FCA to be considered is not the $(1,1,1)-\mathrm{FCA}$ but the $(2,0,2)$ FCA. Of course, one cannot expect the $(1,1,1)$-FCA to be better than MP for heavy traffic since it can only carry a maximum of 3 calls whereas MP can carry 4. In general, any FCA with $M<C a$ cannot be better than MP for heavy traffic.

\section{Acknowledgements}

This work was partially supported by a grant from GTE, AFOSR grant 88-024i, and a grant from Pacific Bell.

\section{References}

[1] Kelly, F. P., "Stochastic Models of Computer Communication Systems," J. R. Statist. Soc. B, vol. 47, no. 3, pp. $379-395,1985$ 